\title{
comsultas téccmicas
}

P. GARCIA DE PAREDES Y GAlBROIS

En contacto con agua freática que contiene

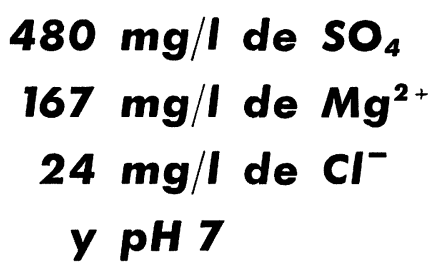

¿se pueden utilizar pilotes de hormigón, compactado por vibración, con $a / c=0,33$, recubrimiento de las armaduras de $25 \mathrm{~mm}$ y como conglomerante cemento P-450?

\section{CONTESTACION}

\section{CALIFICACION DEL AGUA SEGUN VARIAS NORMAS}

I.1. Norma TGL 11 35\%: República Democrática Alemana

Agresividad media debida al contenido de $\mathrm{SO}_{4}=$ y $\mathrm{Mg}^{+}$. La agresividad potencial es un grado mayor (fuertemente agresiva) según la Tabla de Tolerancias, pero disminuye al grado menor por ser mayor de 28 días el tiempo transcurrido entre la fabricación del pilote y el contacto con el medio agresivo.

\section{I.2. Norma DIN 4 030: República Federal Alemana}

Débilmente agresiva por su contenido en $\mathrm{SO}_{4}=\mathrm{y} \mathrm{Mg}^{++}$.

\section{I.3. Norma N 114-45: U.R.S.S.}

Si el agua contiene menos de $1.000 \mathrm{mg} / \mathrm{l}$ de $\mathrm{Mg}^{++}$, sólo se tendrá en cuenta el contenido de sulfatos y cloruros.

Agresiva para hormigones preparados con cemento tipo y clase portland, situados en terrenos con permeabilidad mayor de $10 \mathrm{~m}$ /día y dimensión mínima de 0,5 a $2,5 \mathrm{~m}$; por contener más de $250 \mathrm{mg} / l$ de $\mathrm{SO}_{4}{ }^{2}$ y menos de $1.000 \mathrm{mg} / l \mathrm{de} \mathrm{Cl}^{-}$. 
No es agresiva para hormigones preparados con cementos tipo portland y clase PAS, tipo PUZ (clases PAS-PUZ), tipo siderúrgico, clase PHA por contener menos de $3.000 \mathrm{mg} / l$ de $\mathrm{SO}_{4}{ }^{2}$.

Esta Norma tiene el inconveniente de no fijar un criterio cuantitativo de la influencia destructora. Por ello, V. V. Kind añade unas hojas supletorias aplicables a mayores concentraciones.

Según estas hojas, para hormigones de tipo y clase portland, con: gradiente de presión unilateral del agua/mínima dimensión, menor de 5 (dimensión mínima 0,5 m); con una permeabilidad del terreno comprendida entre 0,1 y $10 \mathrm{~m} /$ día, y una concentración de $\mathrm{SO}_{4}=$ doble de la fijada por la Norma, es decir, de $500 \mathrm{mg} / l$ de $\mathrm{SO}_{4}{ }^{2}$, el agua es ligeramente agresiva. El hormigón de cemento tipo $\mathrm{P}$, clase PAS debe considerarse como si fuese del tipo y clase $\mathrm{P}$ cuando la concentración de $\mathrm{SO}_{4}{ }^{2}$ alcanza $750 \mathrm{mg} / l$ de $\mathrm{SO}_{4}$.

\section{I.4. Building Research Station. Digest 90: Reino Unido}

Grado segundo de agresividad. Contenido de $\mathrm{SO}_{4}{ }^{2}$ comprendido entre 3.000 y $1.200 \mathrm{mg} / \mathrm{l}$ de $\mathrm{SO}_{4}$.

\section{I.5. Asociación Alemana de Fabricantes de Cemento}

Débilmente agresiva por contener menos de $600 \mathrm{mg} / l$ de $\mathrm{SO}_{4}$ y $300 \mathrm{mg} / \mathrm{lde} \mathrm{Mg}^{++}$.

\section{I.6. Manual del Hormigón. Bureau of Reclamation: U.S.A. Dept. Int.}

Positivamente agresiva. (Esta calificación es la inmediata superior a la de agresividad nula y después de ella hay 3 grados más).

Resumen: El agua examinada puede conceptuarse como débilmente agresiva, según la mayoría de las normas, para hormigones preparados con cementos de tipo y clase portland. Con agresividad casi nula para hormigones preparados con cementos de tipo portland, clase PAS; de tipo siderúrgico, clase PHA (mejor si contienen $80 \%$ de escorias y clínker con $4 \%$ de $\mathrm{C}_{3} \mathrm{~A}$ ) y de tipo puzolánico, especialmente con clínker PAS.

\section{RECOMENDACIONES O MEDIDAS DE PROTECCION PARA LOS GRADOS DE AGRESIVIDAD ANTES MENCIONADOS APLICABLES AL AGUA OBJETO DE LA CONSULTA}

\section{II.1. Norma TGL $1135 \%$}

Ante un agua de agresividad media:

El hormigón debe ser como mínimo de la clase B 160, es decir que a los 28 días alcance una resistencia a compresión de $160 \mathrm{kp} / \mathrm{cm}^{2}$, medida sobre probeta cúbica de $20 \mathrm{~cm}$. La relación a/c menor de 0,65 , y contener, como mínimo, $300 \mathrm{~kg}$ de cemento $/ \mathrm{m}^{3}$.

$\mathrm{Si}$ el agua contiene hasta $1.200 \mathrm{mg} / \mathrm{l}$ de $\mathrm{SO}_{4}{ }^{2}$ es apropiado el uso de cementos sulfatoresistentes, tales como: portland con $5 \%$, como máximo, de aluminato tricálcico. Siderúrgicos ricos en escorias: $\mathrm{PHA}$ con clínker que contengan $4 \%$ de $\mathrm{C}_{3} \mathrm{~A}$ como máximo. 


\section{II.2. Norma DIN 4030}

Las recomendaciones de la DVI se formulan en la Norma 1 045, párrafo 6.5.7.3., "Hormigón con alta resistencia a los agresivos químicos".

"La resistencia química del hormigón crece con su compacidad".

El hormigón debe ser tan compacto que la mayor profundidad de penetración del agua, ensayada según DIN 1048, no sobrepase de $5 \mathrm{~cm}$ en ambientes con débil agresividad y 3 $\mathrm{cm}$ si el ambiente es fuertemente agresivo. El coeficiente a/c deberá ser menor que 0,60 si el ataque es débil; y 0,50 si es fuerte.

En débiles agresividades, el contenido de cemento será como mínimo de $350 \mathrm{~kg} / \mathrm{m}^{3}$.

\section{II.3. Norma N 114-45}

Según las tablas adicionales de V. V. Kind, se pueden emplear frente al agua débilmente agresiva $\left(500 \mathrm{mg} / l\right.$ de $\mathrm{SO}_{4}$ ) cementos tipo y clase portland, mejor aún tipo portland, clase PAS, tipo siderúrgico, clase PHA (con clínker PAS) y tipo puzolánico (clínker PAS).

Incrementar, al máximo, la densidad del hormigón mediante reducción de la relación a/c o por tratamiento complejo de vacío y vibración.

Aplicar capas de disolución de silicato sódico y, para prefabricados, tratamiento con gas fluorado (Patente Ocrat).

\section{II.4. Building Research Station. Digest 90}

Para el grado 2 de agresividad que corresponde a un contenido de 360 a $1.440 \mathrm{mg} / \mathrm{l}$ de $\mathrm{SO}_{4}=$ :

Hormigones preparados con cementos del tipo y clase portland o siderúrgicos de la clase PHA: Contenido mínimo $760 \mathrm{~kg} / \mathrm{m}^{3}$ y coeficiente a/c máximo 0,50 en peso.

Hormigones preparados con cementos del tipo portland, clase PAS: contenido mínimo $280 \mathrm{~kg} / \mathrm{m}^{3}$. Coeficiente a/c, máximo 0,55 en peso.

\section{II.5. Asociación de Fabricantes Alemanes de Cemento}

Iguales recomendaciones que la DIN 1045.

\section{II.6. Bureau of Reclamation}

Aconsejable utilizar cemento tipo (A.S.T.M.) II. Portland con $8 \%$ de $\mathrm{C}_{3} \mathrm{~A}$.

Máxima compacidad del hormigón.

\section{R E S U M E N}

1. Máxima compacidad (incluso por tratamiento de vacío). Vibración.

2. Dosificación de cemento: $350 \mathrm{~kg} / \mathrm{m}^{3}$ ó $280 \mathrm{~kg} / \mathrm{m}^{3}$ si se emplea PAS. 
3. Coeficiente a/c: 0,65 (0,55 si se utiliza PAS).

4. Tipos de cemento: P, PAS, PHA (70 \% de escorias y PAS) PUZ (PAS).

\section{CONCLUSIONES}

1) Según los datos suministrados por el consultante el pilote posee las siguientes características favorables para su empleo en contacto con el agua del subsuelo mencionado:

La compactación por vibración.

La resistencia a los 28 días.

La relación a/c. Esta impone una vibración y compactación elevadas.

La naturaleza de los áridos.

2) Los caracteres menos favorables son:

El espesor del recubrimiento.

El conglomerante empleado; si bien dada la compacidad y el curado no es discriminante pues hay normas que lo aceptan sin insistir en los de preparación del hormigón.

Sería deseable conocer la impermeabilidad al agua que poseen los pilotes, pues de ser elevada (como es previsible dada la compactación) desvanecería toda reserva. 


\section{Conglomerante más adecuado para forjados y piezas de hormigón armado en contacto con el agua del mar, pero no sumergidas}

Las sales disueltas en el agua del mar autorizan a temer un efecto corrosivo para el hormigón, más enérgico aún si el contacto con el agua del mar alterna con exposiciones al aire.

Un estudio más completo y, especialmente, los datos recogidos experimentalmente han permitido rectificar el juicio anteriormente expuesto; actualmente se admite que la den sidad o compacidad del hormigón tiene capitalísima importancia.

Para el hormigón no armado se recomiendan los conglomerantes pobres en aluminato tricálcico o aquéllos que contienen, junto al clínker de portland, puzolanas o escorias siderúrgicas, es decir, los de los tipos puzolánico y siderúrgicos del "Pliego" de 1964. Los siderúrgicos conviene sean muy ricos en escorias (1).

Para el hormigón armado, el efecto perjudicial del agua de mar es aún más fuerte y la elección del conglomerante tendrá que satisfacer condiciones casi contradictorias.

Por una parte, todo cuanto asegure un ambiente básico alrededor de las armaduras favorece la inmunidad de éstas; así serán tanto más adecuados los conglomerantes cuanto más hidróxido cálcico liberen durante su hidratación. También el aluminato tricálcico incrementará la defensa de las armaduras, ya que contribuye a fijar los iones cloro y calcio bajo la forma de cloroaluminato (2).

Pero estas dos condiciones favorables para las armaduras no son las más convenientes para asegurar la durabilidad de la pasta hidratada que resiste mejor ante el agua del mar que frente a las disoluciones de sulfatos, precisamente por la presencia del ion cloro tan agresivo para las armaduras.

No se descarta la beneficiosa acción de los recubrimientos superficiales que, si son duración limitada, se pueden emplear en lugares accesibles para renovarlos.

\section{B I B L I O G R A F I A}

(1) I. Biczor.: "Concrete corrosion. Concrete protection” (1964). 4. a edición, págs. 355 a 364.

(2) J. Calleja.: Monografía 256 del I.E.T.c.c. 


\section{Aptitud de un cemento puzolánico (del cual se adjuntan características físicas y químicas) para emplearlo en obras situadas en ambiente maritimo y clima templado}

El ambiente "marítimo", entendiendo por tal las condiciones atmosféricas que existen normalmente hasta la distancia de $500 \mathrm{~m}$ tierra adentro, es más peligroso para el hormigón que el agua del mar.

El hormigón sumergido en el agua del mar se recubre de una capa de carbonato cálcico formado al reaccionar el hidróxido cálcico, liberado durante el endurecimiento de la pasta cementicia, con el carbónico existente en el agua por disolución del anhídrido carbónico del aire y el originado en el ciclo vital de los organismos marinos. Es cierto que esta protección no es absoluta debido a la formación de bicarbonato cálcico soluble, pero, unida a la mayor solubilidad que el ion cloro existente en el mar impone a los sulfoaluminatos cálcicos destructores del hormigón, incrementa la durabilidad del hormigón sumergido.

El hormigón situado en "ambiente marino" sólo tiene como defensa una capacidad tal que impida la penetración de las microscópicas gotitas de agua suspendidas en el aire marítimo. De aquí se deduce que el conglomerante idóneo para el hormigón, situado en las condiciones ya citadas, deberá contribuir a la compacidad del hormigón en la mayor medida posible.

Esta condición la cumplen los conglomerantes puzolánicos (Biczok, "Concrete corrosionconcrete protection", ed. 1972).

En las puzolanas es esencial su capacidad de formar combinaciones cálcicas de propiedades hidráulicas a temperaturas medianas. Estas combinaciones, a las cuales se ha concedido por muchos años la principal ventaja en el uso de los conglomerantes puzolánicos, en realidad, según Steopoe, son transitorias y el verdadero valor a efectos de durabilidad reside en que se hidrolizan originando geles voluminosos de alúmina y de sílice que, además de contribuir a la cohesión (traducible en resistencias mecánicas), obturan los poros incrementando la compacidad del hormigón. Esta cualidad les confiere una indiscutible idoneidad para la preparación de hormigones "marítimos".

La información que el consultante ha remitido, y que se incluye en esta "consulta técnica", permite esperar una excelente durabilidad de los hormigones preparados con el conglomerante que posee las características químicas, físicas y mecánicas consignadas en los informes que remitió. 


\section{Conglomerante más idóneo para resistir el ataque de un agua cuyo análisis se conoce}

El agua que estará en contacto con el hormigón, es, según el informe que remite el peticionario, "honestamente agresiva"; la concentración de $\mathrm{SO}_{4}{ }^{2}$ es de $315 \mathrm{mg} / l$.

"La Building Research Station (G. B.) en su "Digest" 90 (2. ${ }^{a}$ serie) (1968) incluiría este agua en el límite entre las clases 1 (300 p.p.m. $\left.\mathrm{SO}_{3}\right)$ y 2 (300 a 12.000 p.p.m. $\mathrm{SO}_{3}$ ). En estos casos recomienda para el hormigón "denso y compactado al máximo", utilizar:

a) Cemento portland ordinario o cemento portland de alto horno con más de $60 \%$ de escorias, en cantidad mínima de $380 \mathrm{~kg} / \mathrm{m}^{3}$ y máximo factor a/c 0,50 ponderal.

b) Cemento portland sulfatorresistente (máximo 3,5 \% de $\mathrm{C}_{3} \mathrm{~A}$ ) en cantidad mínima de $280 \mathrm{~kg} / \mathrm{m}^{3}$, máximo a/c $=0,55 \%$ en peso.

c) Cemento supersulfatado (5\% máximo, portland).

La norma DIN 1045 "Control y preparación del hormigón" establece en el párrafo 5.2.4.3. "Ataque por sulfatos y agua de mar" lo siguiente: "Independientemente de la calificación que cada caso merezca según las posibilidades de ataque, el hormigón debe siempre prepararse con cementos de alta resistencia a los sulfatos cuando el agua contenga más de $400 \mathrm{mg}$ de $\mathrm{SO}_{4}=$ por litro o el terreno más de $3.000 \mathrm{mg}$ de $\mathrm{SO}_{4}{ }^{~}$ por kilo de tierra seca".

El cemento portland contendrá, como máximo, $3 \%$ de aluminato tricálcico (calculado) y $5 \%$ de $\mathrm{Al}_{2} \mathrm{O}_{3}$.

Los cementos siderúrgicos, ricos en escorias, contendrán de 70 a $80 \%$ de escoria básica granulada.

En la Unión Soviética, los cementos utilizabìes ante un posible ataque por sulfatos están clasificados en el siguiente orden decreciente de durabilidad:

1. Cementos siderúrgicos y puzolano-resistentes a los sulfatos.

2..$^{\circ}$ Cemento portland resistente a los sulfatos.

3. ${ }^{\circ}$ Cemento portland.

Los cementos del grupo primero deben contener clínker de portland con menos del $8 \%$ de aluminato tricálcico.

La posible variedad de escorias obtenidas en el horno alto ha dado lugar a numerosos estudios sobre la sulfatodurabilidad de los cementos siderúrgicos.

Según I. Biczok, (Concrete corrosion-concrete protection), los cementos siderúrgicos de horno alto poseen un alto grado de estabilidad frente a los efectos agresivos, en primer lugar por ser menor la cantidad de hidróxido cálcico que liberan en su hidratación y además por un efecto calcio-fijador ejercido por las escorias. La menor alcalinidad da lugar a la formación de hidro sílico-aluminatos de calcio sulfatorresistentes. Según V. V. Kind, además se forman sulfoaluminatos no cristalinos; esto da lugar a que los cementos con una proporción de escorias mayor del $60 \%$ son altamente resistentes, incluso en disoluciones de sulfato sódico concentradas. 
(Esta opinión de V. V. Kind se ha comprobado experimentalmente en el Instituto Eduardo Torroja aplicando el método de ensayo de Koch y Steinegger y el de Merriman modificado en el Instituto).

En el V Simposio Internacional sobre el cemento y el hormigón, celebrado en Tokio (1968), F. Schröder y H. G. Smolczyk informan del siguiente modo:

"Existe una amplia literatura sobre el comportamiento de los cementos siderúrgicos en contacto con disoluciones agresivas, especialmente de sulfatos y con el agua de mar".

"La gran mayoría de todos los ensayos de laboratorio han puesto de manifiesto una estabilidad satisfactoria de los cementos de horno alto, ricos en escorias, cuando se hallan en contacto con disoluciones de sulfatos, de cloruros, aguas ácidas y agua de mar".

Los ensayos, a escala real, a largo plazo con cementos siderúrgicos han demostrado un comportamiento más favorable de los ricos en escorias que, sin excepción, acusaron una mejor durabilidad.

A. Eckhardt, W. Kronsbein, K. Wesche y A. Hummel dedujeron de la observación de hormigones expuestos durante 25 años en agua de mar (Mar del Norte), el siguiente orden creciente de resistencia química:

1) Cemento portland (10 a $13 \%$ de $\left.\mathrm{C}_{3} \mathrm{~A}\right)$.

2) Cemento portland férrico (25 a $35 \%$ de escorias).

3) Cemento de escorias (50\% y más de $60 \%$ de escorias).

4) Cemento aluminoso.

En Noruega se realizó un estudio con pilotes de hormigón armado que permanecieron 27 años en el mar. El hormigón con cemento siderúrgico (más de $60 \%$ de escorias) y un coeficiente $\mathrm{a} / \mathrm{c}=0,66$, así como el preparado con cemento portland (menos de $3 \%$ de $\mathrm{C}_{3} \mathrm{~A}$ ) y un coeficiente $\mathrm{a} / \mathrm{c}=0,56$ aparecieron con gran estabilidad, en tanto los pilotes de hormigón preparado con portland $\left(11 \%\right.$ de $\left.\mathrm{C}_{3} \mathrm{~A}\right)$ y a/c $=0,61$ se habían destruido.

En la discusión de este informe, E. Spoh hizo notar que: "No todos los cementos de escorias son resistentes a los sulfatos".

"Tiene importancia decisiva el tipo de escorias". "Escorias con un contenido de $\mathrm{Al}_{2} \mathrm{O}_{3}$, alrededor del $11 \%$ (ponderal) proporcionan, cuando se las moltura con un clínker de portland, en la proporción de $60 \%$ de escorias y $40 \%$ de portland, cementos con igual resistencia química a los sulfatos que los del tipo V de la A.S.T.M. (Cementos resistentes a los sulfatos)".

A juicio de Smolczyk "No existen métodos eficaces para el ensayo acelerado de la sulfatorresistencia de los conglomerantes; la experiencia ofrecida al cabo de muchos años, le autoriza a creer que es más acertado recoger la experiencia dada por las estructuras de hormigón situadas en ambientes agresivos".

"Las numerosas estructuras costeras de Francia y Bélgica realizadas con cementos siderúrgicos poseen alta resistencia al ataque químico. Los cementos portland pobres o exentos de aluminato tricálcico son muy resistentes al ataque químico. Los conglomerantes siderúrgicos, y especialmente aquellos ricos en escorias, además de contener poco trialumi-nato (por ser menor la cantidad de clínker que contienen), originan durante su hidratación abundante gel silícico que se combina con los componentes alumínicos y cálcicos formando los hidrogranates específicamente sulfatorresistentes". 
W. Kramer, del Instituto aleman de investigaciones sobre escorias siderúrgicas, en Rheinhausen, en el IV Simposio sobre el cemento y el hormigón, celebrado en Wáshington el año 1960, informó de lo siguiente:

"Las escorias siderúrgicas, en contacto con el agua ceden a ella iones calcio ; sobre los granos de escoria se deposita una película de hidratos y geles impermeables de hidróxido alumínico que, a su vez, se disuelven cuando la concentración de ion calcio alcanza determinados valores, por ello la cesión de calcio es continua". "La formación de hidrogranates es dependiente de la concentración de calcio y se favorece cuando el líquido que empapa la pasta del cemento se convierte en disolución sobresaturada de calcio, lo que ocurre en los conglomerantes siderúrgicos".

H. O. Daniel puso de manifiesto la formación de enlaces entre átomos de silicio con oxígeno y $\mathrm{OH}$. Este tipo de enlaces es de excepcional importancia en la estructura del conglomerante siderúrgico hidratado, por su alta resistencia a las acciones químicas. Los ensayos llevados a cabo en el Instituto de Investigaciones sobre escorias, en Rheinhausen, han demostrado que en los cementos siderúrgicos de bajo contenido de clínker se fijan gran cantidad de $\mathrm{OH}^{-}$; esto explica la satisfactoria estabilidad de los cementos de esta clase frente a las influencias de los agresivos químicos. 